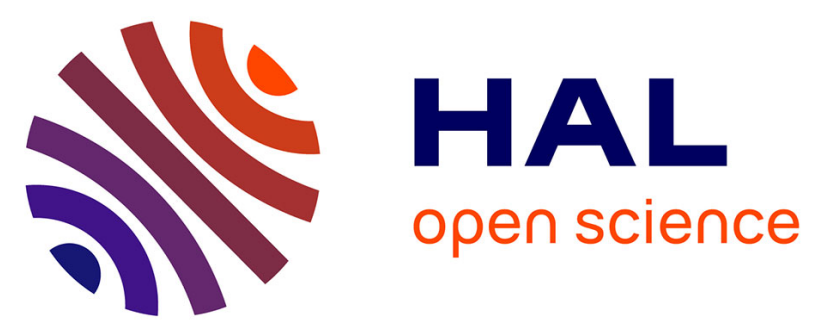

\title{
HandiViz project: clinical validation of a driving assistance for electrical wheelchair
}

Marie Babel, François Pasteau, Sylvain Guégan, Philippe Gallien, Benoît

Nicolas, Bastien Fraudet, Sophie Achille-Fauveau, Daniel Guillard

\section{To cite this version:}

Marie Babel, François Pasteau, Sylvain Guégan, Philippe Gallien, Benoît Nicolas, et al.. HandiViz project: clinical validation of a driving assistance for electrical wheelchair. IEEE Workshop On Advanced Robotics And Its Social Impacts (ARSO), Jul 2015, Lyon, France. 10.1109/ARSO.2015.7428213 . hal-01175784

\section{HAL Id: hal-01175784 \\ https://hal.science/hal-01175784}

Submitted on 12 Jul 2015

HAL is a multi-disciplinary open access archive for the deposit and dissemination of scientific research documents, whether they are published or not. The documents may come from teaching and research institutions in France or abroad, or from public or private research centers.
L'archive ouverte pluridisciplinaire HAL, est destinée au dépôt et à la diffusion de documents scientifiques de niveau recherche, publiés ou non, émanant des établissements d'enseignement et de recherche français ou étrangers, des laboratoires publics ou privés. 


\title{
HandiViz project: clinical validation of a driving assistance for electrical wheelchair
}

\author{
Marie Babel $^{1}$, François Pasteau ${ }^{1}$, Sylvain Guégan ${ }^{2}$, \\ Philippe Gallien ${ }^{3}$, Benoît Nicolas ${ }^{3}$, Bastien Fraudet ${ }^{3}$, \\ Sophie Achille-Fauveau ${ }^{4}$, Daniel Guillard ${ }^{5}$
}

\begin{abstract}
Autonomy and independence in daily life, whatever the impairment of mobility, constitute fundamental needs that participate to the self-esteem and the well-being of disabled people. In this context, assistive technologies are a relevant answer. To address the driving assistance issue, we propose in this paper a unified shared control framework able to smoothly correct the trajectory of the electrical wheelchair. The system integrates the manual control with sensor-based constraints by means of a dedicated optimization strategy. The resulting low-complex and low-cost embedded system is easily plugged onto on-the-shelf wheelchairs. The robotic solution has been then validated through clinical trials that have been conducted within the Rehabilitation Center of Pôle Saint Hélier (France) with 25 volunteering patients presenting different disabling neuro-pathologies. This assistive tool is shown to be intuitive and robust as it respects the user intention, it does not alter perception while reducing the number of collisions in case of hazardous maneuvers or in crowded environment.
\end{abstract}

\section{INTRODUCTION}

Global population ageing as well as disability compensation constitute major challenging societal and economic issues [1]. In particular, achieving autonomy remains a fundamental need that contributes to the wellness and the well-being. In this context, innovative and smart technologies are designed to achieve independance while matching user individual wants [2].

Assistive technologies are then considered as a great opportunity to improve the quality of life. Sensorial, physical and mental limitations can be exceeded by enhancing existing abilities. Assistive robots can then be seen as a solution for realizing daily living tasks.

For people suffering of motor disabilities (due to coordination limitations, dexterity incapacities, injury, accidents...), electrical wheelchair remains one of the most used assistive technology equipment as it is synonym of freedom of navigation and travel [3]. However, operating a wheechair in a secure way requires cognitive skills (typically to anticipate obstacles and to plan a safe trajectory) as well as performant visual-perceptual abilities [4]. As a consequence,

\footnotetext{
*This work was not supported by any organization

${ }^{1}$ Marie Babel and François Pasteau with the Lagadic Team at IRISA/Inria Rennes and INSA Rennes, France marie.babel@irisa.fr

${ }^{2}$ Sylvain Guégan is with the LGCGM at INSA Rennes, France

${ }^{3}$ Philippe Gallien, Benoit Nicolas and Bastien Fraudet are with the Rehabilitation Center Ple Saint Hélier Rennes, France

${ }^{4}$ Sophie Achille-Fauveau is with the Healthcare Network Breizh Paralysie Cérébrale Rennes, France

${ }^{4}$ Daniel Guillard is with Ergovie Rennes, France
}

because of inadequate and dangerous reactions encountered while navigating, some disabled people are not allowed to drive electrical wheelchair, thus dramatically reducing their autonomy [5]. Therefore, designing a robotic assistive solution related to wheelchair navigation remains of major importance.

Recent advanced studies then deal with Smart Wheelchairs in order to improve user quality of experience. As for example, the NavChair [6], the European FP7 Radhar project [7] and the recent SYSIASS [8] and COALAS projects [9] were able to design systems that take partial or full control from the user for safe and effective navigation assistance. However, the main difficulty related to Smart Wheelchair systems is to associate low-cost embedded solutions and efficient and robust framework [10].

The French HandiViz project aims then at realizing an intuitive and low-cost driving assistance. The idea is to progressively correct the trajectory in a smooth manner so that to avoid static or dynamic obstacles. The related robotic system should be easily plugged onto any off-theshelf wheelchairs thus targeting a widespread usage as a commercialization of the assistance system is envisaged.

To this aim, different control frameworks based on a shared control strategy have been previously developped. However, in the literature, such robotic assistance systems typically require expensive multi-sensor system (e.g. laserrange finder [11], [12]), specific instrumentation or adapted interfaces [13], [14]. In this paper, we design a generic shared control system that is independant of the used sensor types, as soon as these sensors are able to provide a distance information. The proposed solution fuses then the user input with a dedicated control law without any a priori knowledge of the environment, in order to design a reactive local approach. In this domain, state-of-the-art techniques typically use visual servoing frameworks [15], [16] or Potential Field Methods [17], [12] that act as repulsion forces. In our case, contrary to [12] that abruptly switches to obstacle avoidance/full control states, we define a unified framework able to progressively and adaptively modulate the applied trajectory correction with respect to the distance with the detected obstacles. It results in a smooth correction thus improving the quality of navigation.

Besides, assessing a robotic system remains a critical step in the development of an assistive technology. As disability is a complex notion, it is difficult to provide a universal bench- 
mark that is convenient for any situation [18]. Moreover, because clinical trials are subject to the recommendation of ethics committees, we first have to validate both the robustness and the acceptability of the solution before proposing the assistive solution to patients in great difficulty. Hence we here propose a first step in the validation process and we elaborate tests along an ecological circuit with volunteering patients who use their electrical wheelchair regularly. The objective is then to measure the ability of the system to avoid collisions without modifying user habits and sensations. To this aim, we put together an interdisciplinary team composed of roboticians, physiotherapists, occupational therapists and rehabilitation medicine specialists.

This paper is organized as follows: Section II describes the generic shared control algorithm that is independant of the sensors used. Section III determines first the experimental setup. In particular, the robotized wheelchair used for the tests with patients is only equipped with low-cost ultrasound sensors. Then we present the benchmarking methodology and discuss the clinical trials and the related results that have been obtained in the Rehabilitation Center of Pôle Saint Hélier in Rennes (France) with the help of 23 patients.

\section{GENERIC SHARED CONTROL APPROACH}

Designing a trajectory correction process requires shared control frameworks. The resulting semi-autonomous navigation system fuses manual control and robotic control, leading to a man-in-the-loop robotics application. To increase the Quality of Experience of such an assisted navigation, the proposed solution should integrate the user intention. To this aim, we here propose a generic shared control approach that takes into account the user velocity instruction.

\section{A. Modelling}

As shown on Fig.1,

- let $\mathbf{u}=(u, \omega)$ be the velocity of the wheelchair,

- let $\mathbf{v}_{c_{i}}=\left(\boldsymbol{u}_{c_{i}}, \boldsymbol{\omega}_{c_{i}}\right)$ be the velocity of the sensor $c_{i}$,

- let $x_{i}$ be the distance from the sensor $c_{i}$ to the obstacle,

- let $x_{i}^{*}$ be a minimum distance from the sensor $c_{i}$ to the obstacle,

- let $e_{c_{i}}=x_{i}-x_{i}^{*}$ be the error between $x_{i}$ and $x_{i}^{*}$.

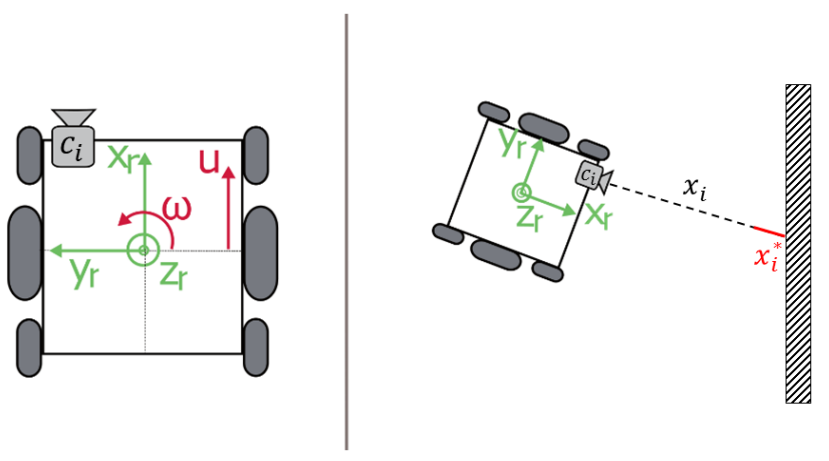

Fig. 1. Definition of the robot frame

We can define a Jacobian $\mathbf{J}$ such as

$$
\mathbf{v}_{c_{i}}=\mathbf{J u}
$$

We obtain

$$
\mathbf{v}_{c_{i}}=\mathbf{J}_{u} u+\mathbf{J}_{\omega} \omega
$$

with $\mathbf{J}_{u}$ of size $6 \times 1$ and $\mathbf{J}_{\omega}$ of size $6 \times 1$

- Let $\mathbf{L}_{x_{i}}$ be the interaction matrix for the sensor $c_{i}$.

We have

$$
\dot{x_{i}}=\mathbf{L}_{x_{i}} \mathbf{v}_{c_{i}}
$$

with $\mathbf{L}_{x_{i}}$ of size $1 \times 6$

By combining equations (2) and (3), we obtain

$$
\dot{x_{i}}=\mathbf{L}_{x_{i}} \mathbf{J}_{u} u+\mathbf{L}_{x_{i}} \mathbf{J}_{\omega} \omega
$$

To avoid collision with the obstacle, we constrain $\dot{x_{i}}$ by a minimum value $-\lambda e_{c_{i}}$ corresponding to a proportional corrector. Consequently, we get

$$
\dot{x_{i}} \geq-\lambda e_{c_{i}}
$$

By combining equation (4) and inequation (5), we get

$$
\mathbf{L}_{x_{i}} \mathbf{J}_{u} u+\mathbf{L}_{x_{i}} \mathbf{J}_{\omega} \omega \geq-\lambda e_{c_{i}}
$$

As $\mathbf{J}_{u}$ is of size $6 \times 1$ and $\mathbf{L}_{x_{i}}$ is of size $1 \times 6$, we can define a scalar $a_{c_{i}}$ such as

$$
a_{c_{i}}=\mathbf{L}_{x_{i}} \mathbf{J}_{u}
$$

Similarly, we can define a scalar $b_{c_{i}}$ such as

$$
b_{c_{i}}=\mathbf{L}_{x_{i}} \mathbf{J}_{\omega}
$$

Then, we get

$$
a_{c_{i}} u+b_{c_{i}} \omega \geq-\lambda e_{c_{i}}
$$

which defines a half-plane in the $u-\omega$ plane as shown on Fig.2.

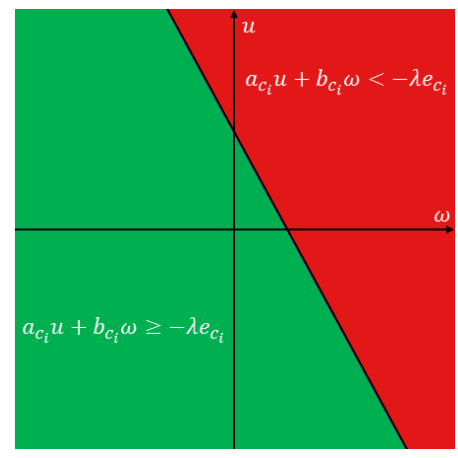

Fig. 2. Definition of half plan in the $u-\omega$ plane

Inequation (9) can be rewritten as

$$
\text { Au } \geq \mathbf{B}
$$

with $\mathbf{A}=\left[\begin{array}{ll}a_{c_{i}} & b_{c_{i}}\end{array}\right]$ and $\mathbf{B}=\left[-\lambda e_{c_{i}}\right]$.

When considering $\mathrm{N}$ sensors, we can rewrite inequation (10) using

$$
\mathbf{A}=\left[\begin{array}{cc}
a_{c_{0}} & b_{c_{0}} \\
a_{c_{1}} & b_{c_{1}} \\
\cdots & \cdots \\
a_{c_{N-1}} & b_{c_{N-1}}
\end{array}\right] \text { and } \mathbf{B}=\left[\begin{array}{c}
-\lambda e_{c_{0}} \\
-\lambda e_{c_{1}} \\
\cdots \\
-\lambda e_{c_{N-1}}
\end{array}\right] \text {. }
$$




\section{B. Computing control values}

- Let $\mathbf{u}_{o p}=\left(u_{o p}, \omega_{o p}\right)$ be the velocity input from the user,

- Let $\mathbf{u}_{c m d}=\left(u_{c m d}, \omega_{c m d}\right)$ be the velocity sent to the robot.

$\mathbf{u}_{c m d}$ is computed from $\mathbf{u}_{o p}$ under constraints (10) by minimizing a cost function $f$. This can written as an optimization problem using

$$
\left\{\begin{aligned}
\mathbf{u}_{c m d} & =\min _{\mathbf{u}} f\left(\mathbf{u}_{o p}, \mathbf{u}\right) \\
\mathbf{A u} & \geq \mathbf{B}
\end{aligned}\right.
$$

We define the function $f$ such as

$$
f\left(\mathbf{u}_{o p}, \mathbf{u}\right)=\alpha\left(u-u_{o p}\right)^{2}+\beta\left(\omega-\omega_{o p}\right)^{2}
$$

with $\alpha \geq 0$ and $\beta \geq 0$.

Then the problem can be written as a classical quadratic optimization problem using

$$
\left\{\begin{aligned}
\mathbf{u}_{c m d} & =\min _{\mathbf{u}} \mathbf{g}^{T} \mathbf{u}+\frac{1}{2} \mathbf{u}^{T} \mathbf{H u} \\
\mathbf{A u} & \geq \mathbf{B}
\end{aligned}\right.
$$

with $\mathbf{g}=\mathbf{u}_{o p}{ }^{T}\left[\begin{array}{cc}-\alpha & 0 \\ 0 & -\beta\end{array}\right]$ and $\mathbf{H}=\left[\begin{array}{cc}\alpha & 0 \\ 0 & \beta\end{array}\right]$.

As shown on Fig.3, we can define two sets $\mathbf{P}_{0}$ and $\mathbf{P}_{1}$ such as

$$
\begin{aligned}
& \mathbf{P}_{0}=\quad\{\mathbf{u} \mid \mathbf{A u} \geq \mathbf{B}\} \\
& \mathbf{P}_{1}=\{\mathbf{u} \mid \mathbf{A u}>\mathbf{B}\} .
\end{aligned}
$$

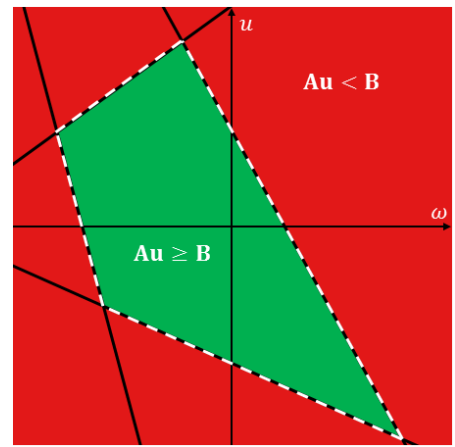

\section{$\mathrm{P}_{0}$}

\section{$\mathbf{P}_{1}$}

$1-\overline{\mathbf{P}_{0}-\mathbf{P}_{1}}$ $\mid \mathbf{P}_{0}-\mathbf{P}_{1}$

Fig. 3. Definition of $\mathbf{P}_{0}$ and $\mathbf{P}_{1}$

Hence the problem (13) can be resolved only if $\mathbf{P}_{0} \neq \emptyset$. If $\mathbf{P}_{0}=\emptyset, x_{i}^{*}$ can be dynamically decreased to expand the size of $\mathbf{P}_{0}$.

In fact, the quadratic optimization problem (13) can be solved by observing two different cases:

- Case 1: if $\mathbf{u}_{o p} \in \mathbf{P}_{0}$, then $\mathbf{u}_{c m d}=\mathbf{u}_{o p}$,

- Case 2: otherwise, as the cost function is convex and its minimum is reached when $\mathbf{u}=\mathbf{u}_{o p}$, $\mathbf{u}_{c m d} \in \mathbf{P}_{0}-\mathbf{P}_{1}$ meaning that it exists at least one $i$ such as $a_{c_{i}} u+b_{c_{i}} \omega=-\lambda e_{c_{i}}$.

A search along $\mathbf{P}_{0}-\mathbf{P}_{1}$ gives the result of the optimisation problem 13.
To sum up, the proposed shared control solution allows the user to control the wheelchair while observing safety constraints. The formulation of the problem leads to solve a simple quadratic system under constraints. The resulting algorithm provides then a progressive trajectory correction as no singularities can be observed.

Next section is devoted to the clinical trials and the evaluation of this assistive tool.

\section{ClinicAl tRials}

Clinical trials are mandatory when considering robotic assistance device for wheelchair navigation purposes. This study constitutes the first step in clinical validation. Before envisaging a widespread usage of the system, we have to first demonstrate the robustness and the acceptability of the solution, along with its ability to reduce collision.

This evaluation study has received a favorable opinion from the ethics committee of Pontchaillou Hospital (Rennes, France)

\section{A. Experimental setup}

The wheelchair used in the clinical trials is based on an off-the-shelf YouQ Luca wheelchair. This wheelchair has 5 wheels, where 2 of which are actuated and the 3 others are caster wheels. The user joystick and the R-Net wheelchair electronics come from Penny \& Giles.

In Section II we described a generic shared control process which has been designed independently of the sensors used. To cope with the low-cost requirement that should guarantee in the end a widespread usage, the wheelchair has been equipped with 15 ultrasound sensors installed all around it, as shown on Fig. 4.

When considering the hardware architecture, an ARM CPU board is embedded onto the wheelchair to run the actual algorithm. With this setup, a latency of less than $10 \mathrm{~ms}$ is to be expected due to the nature of the communications encountered on the R-Net CAN bus. This latency has been shown to be undetectable by wheelchair users.

A user interface is installed behind the wheelchair: it allows the clinicians to start/stop the experiments and enable or disable the assistance. The volunteers are then not aware of the activation or not of the assistance module.

\section{B. Evaluation methodology}

The objective of this study is to validate the robustness and the efficiency of the system, as well as this acceptability. Hence we have recruited 23 disabled people who are considered as experts in wheelchair driving. These patients present different pathologies that induce different degrees in motor impairments: tetraplegia, cerebral palsy, amputee, brain injured, stroke. In order to avoid a bias in the evaluation process, we consider only one configuration of the system: users perform then in the same conditions. Then the parametrisation of the wheelchair is realized only once, which can slightly differs from the parametrisation of the wheelchair of each participant. In addition, the maximum speed is set to $0.8 \mathrm{~m} / \mathrm{s}$ for all the volunteers. 


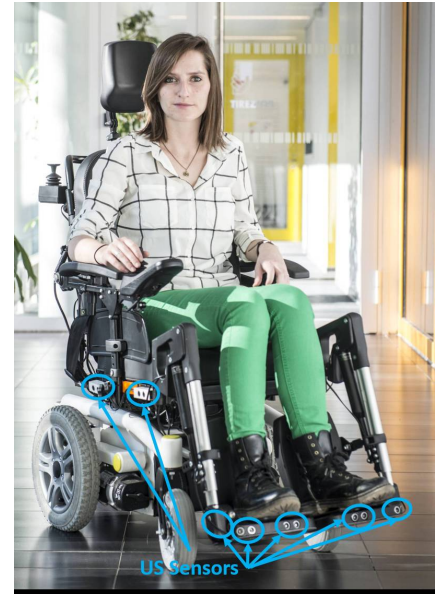

Fig. 4. Robotized wheelchair equipped with ultrasound sensors located on the side and on the rear of the body, as well as in footplates.

Tests have been performed at Rehabilitation Center Pôle Saint Hélier in Rennes, France. To this aim, an ecological circuit has been defined: Fig. 5 shows the complete path to be achieved by the users. The navigation is realized in real conditions, meaning that the corridors are crowded, and that dynamic or static obstacles are disposed along the circuit. In addition, users were supposed to realize difficult maneuvers in narrow spaces (turning around, rolling back at the end in a small corridor).

At the beginning of the experiments, volunteers were asked to reach the therapeutic apartment, then going back the same way until reaching a narrow corridor where they have to realize a 90 rotation before entering backwards. We dispose no landmark on the floor or on the walls. Volunteers receive no further indications so that they could choose the trajectory they want.

Random double-blind trials were then conducted: for each volunteer, experiments were performed twice with and without assistance without the patient knowing which one is which. Each participant took about four minutes to complete each trial. A short QUEST-like questionnaire was fulfill at the end of each trial [19]. .

\section{Results}

Table I sums up the results from the clinical trials as described in the previous section. For each participant, we first determine whether the first experiment is realized with or without assistance. Then in each case, the number of collisions are recorded by an external observer and the QUEST questionnaire is fulfilled, leading to a score between 0 and 40.

In addition, when the assistance module is activated, the activation ratio, corresponding to the percentage of navigating time during which the correction is applied, is measured.

We can observed that on average, the number of collisions when assistance is engaged is reduced by half when compared to navigation without assistance. Moreover, as the answers to the questionnaire are in favour of the assisted trial, it exhibits the acceptability of the assistance solution.
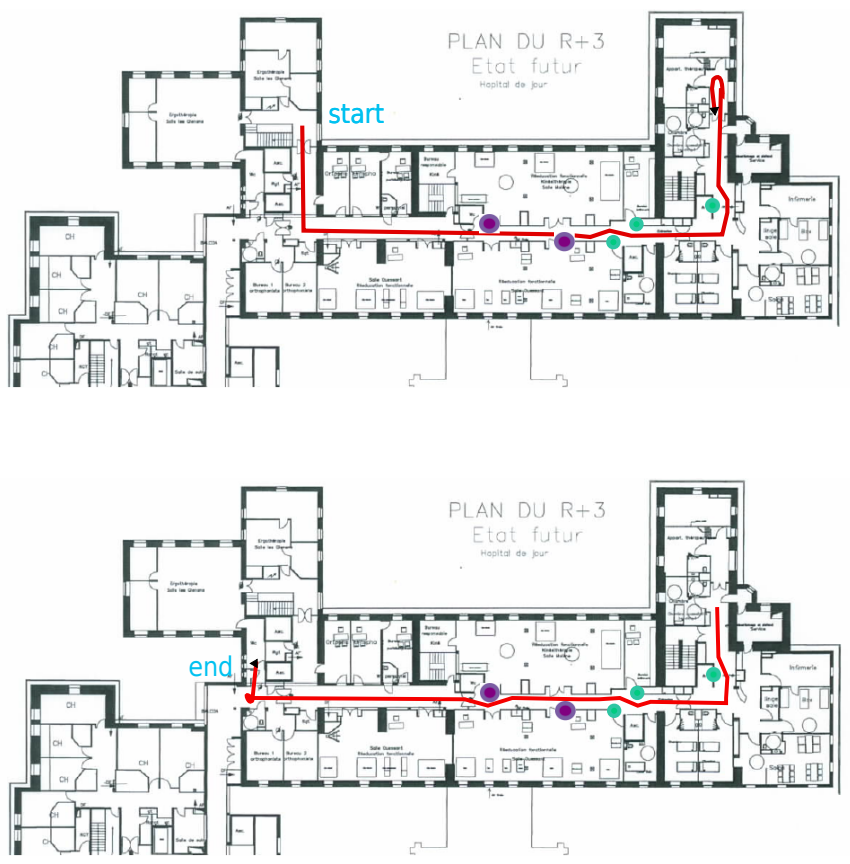

Fig. 5. Ecological circuit

In particular, it emphasizes the fact that the assistance does not alter the driving experience even if the activation ratio can be up to $35.51 \%$.

Collisions typically occur during difficult maneuvers as illustrated in Table II, for example while reversing in a narrow corridor or crossing a moving obstacle or operating a sharp turn around small static obstacles that could be difficult to perceive for people suffering of visual impairments.

\section{CONCLUSION}

This paper has presented a unified shared control framework able to provide a smooth trajectory correction while driving a wheelchair. Based on an efficient fusion of the user input and the obstacle avoidance task, a progressive modulation of the velocity of the wheelchair is obtained.

Clinical trials involving 23 volunteering disabled people have shown that for individuals who are used to drive their electrical wheelchair daily, the driving assistance is intuitive and does not modify their perception. In addition, while navigating along an ecological circuit, collisions during difficult maneuvers (turning around in a narrow space, rolling backward...) are reduced when the assistance is engaged.

Thus this paper demonstrates the clinical validation of the proposed assistive system. The next step in the clinical validation process will consist in realizing new trials with disabled who experience great driving difficulties or are even not allowed to drive an electrical wheelchair because of their poor navigation skills.

\section{ACKNOWLEDGEMENT}

This work has been partially supported by the SATT Ouest Valorisation. The authors would like to thank Geoffroy Henry 


\begin{tabular}{|c|c|c|c|c|c|c|}
\hline \multirow{2}{*}{ Participant } & \multirow{2}{*}{ First } & \multicolumn{2}{|c|}{ Without Assistance } & \multicolumn{3}{|c|}{ With Assistance } \\
\hline & & Collisions & Quest & Collisions & Quest & $\begin{array}{c}\text { Activation } \\
\text { rate }(\%)\end{array}$ \\
\hline 1 & With & $\mathbf{0}$ & 31 & 1 & 30 & 30.92 \\
\hline 2 & Without & 5 & 29 & 2 & 31 & 12.37 \\
\hline 3 & With & 1 & 32 & $\mathbf{0}$ & 37 & 10.95 \\
\hline 4 & With & $\mathbf{0}$ & 28 & $\mathbf{0}$ & 32 & 3.04 \\
\hline 5 & With & 2 & 31 & $\mathbf{0}$ & 31 & 12.46 \\
\hline 6 & With & $\mathbf{0}$ & 32 & $\mathbf{0}$ & 34 & 18.09 \\
\hline 7 & Without & $\mathbf{0}$ & 31 & $\mathbf{0}$ & 31 & 35.51 \\
\hline 8 & Without & 2 & 36 & $\mathbf{0}$ & 35 & 15.52 \\
\hline 9 & With & 1 & 36 & $\mathbf{0}$ & 37 & 5.32 \\
\hline 10 & Without & 2 & 30 & 3 & 31 & 4.84 \\
\hline 11 & With & 1 & 36 & 3 & 35 & 3.87 \\
\hline 12 & Without & $\mathbf{0}$ & 40 & 0 & 40 & 2.12 \\
\hline 13 & With & 1 & 31 & 0 & 31 & 4.19 \\
\hline 14 & Without & $\mathbf{0}$ & 36 & 0 & 36 & 4.67 \\
\hline 15 & With & 1 & 32 & 0 & 32 & 0.60 \\
\hline 16 & Without & 1 & 26 & 0 & 25 & 2.46 \\
\hline 17 & With & 3 & 36 & 0 & 36 & 4.02 \\
\hline 18 & Without & 0 & 33 & 1 & 36 & 4.28 \\
\hline 19 & Without & 0 & 34 & 0 & 33 & 0.60 \\
\hline 20 & Without & 0 & 36 & 0 & 36 & 2.03 \\
\hline 21 & With & 0 & 34 & $\mathbf{0}$ & 34 & 4.51 \\
\hline 22 & With & 0 & 33 & 0 & 32 & 2.69 \\
\hline \multirow[t]{2}{*}{23} & Without & 0 & 34 & 0 & 31 & 1.48 \\
\hline & Average & 0.869 & 32,91 & 0.435 & 33,30 & 8.11 \\
\hline
\end{tabular}

TABLE I

COLLISION RESULTS

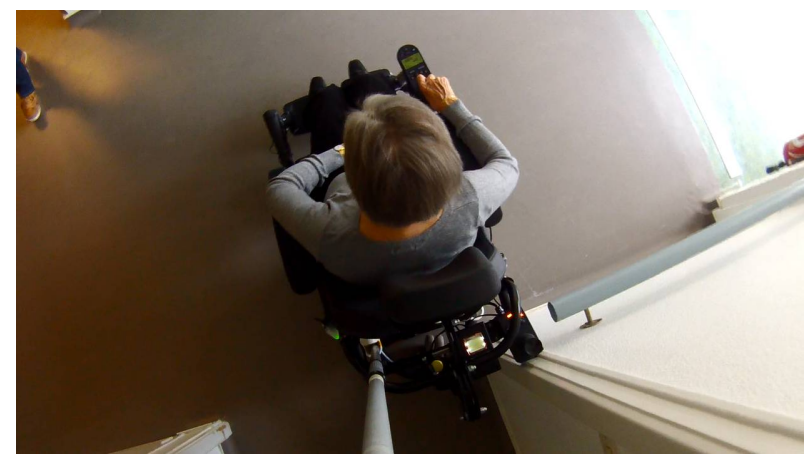

a) Collision while reversing

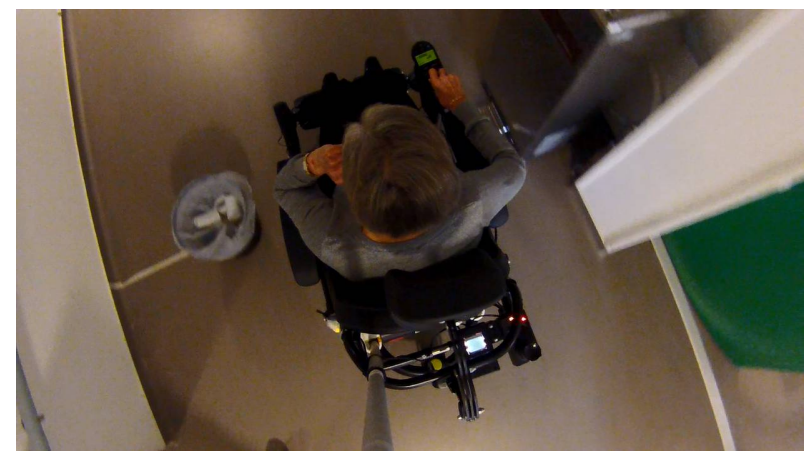

c) Rotation around a dust bin with assistance - No Collision

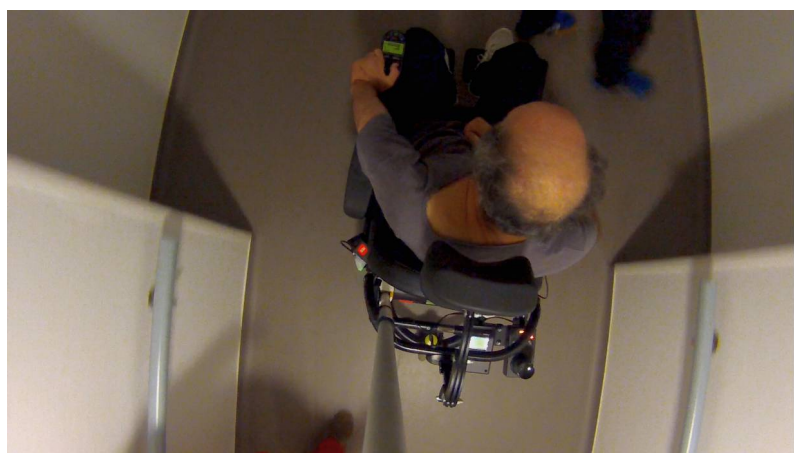

b) Moving obstacle

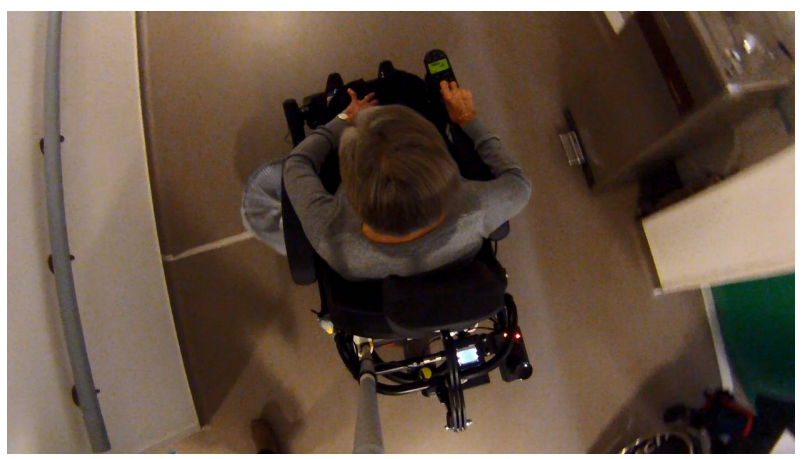

d) Rotation around a dust bin without assistance - 1 Collision

TABLE II

IMAGES FROM THE EXPERIMENTS 
and Luc Le Pape from Ergovie, Eric Bazin from INSA

Rennes, Amélie Colin from Healthcare Network Breizh Paralysie Cérébrale and all the volunteers who contributed to this study.

\section{REFERENCES}

[1] J. Beard, S. Biggs, D. E. Bloom, L. P. Fried, P. R. Hogan, A. Kalache, and S. J. Olshansky, "Global Population Ageing: Peril or Promise?" Program on the Global Demography of Aging, Tech. Rep. 8912, Jan. 2012.

[2] A. Helal, M. Mokhtari, and B. Abdulrazak, The engineering handbook of smart technology for aging, disability, and independence. Wiley, 2008.

[3] F. Routhier, C. Vincent, J. Desrosiers, and S. Nadeau, "Mobility of wheelchair users: a proposed performance assessment framework," Disability \& Rehabilitation, vol. 25, no. 1, pp. 19-34, 2003.

[4] R. H. L. Wang, "Enabling power wheelchair mobility with long-term care home residents with cognitive impairments," Ph.D. dissertation, University of Toronto, 2011.

[5] R. H. Wang, P. J. Holliday, and G. R. Fernie, "Power mobility for a nursing home resident with dementia," American Journal of Occupational Therapy, vol. 63, no. 6, pp. 765-771, 2009.

[6] S. P. Levine, D. A. Bell, L. A. Jaros, R. C. Simpson, Y. Koren, and J. Borenstein, "The navchair assistive wheelchair navigation system," IEEE Transactions on Rehabilitation Engineering, vol. 7, pp. 443-451, 1999.

[7] E. Demeester, E. EB Vander Poorten, A. Hüntemann, and J. De Schutter, "Wheelchair navigation assistance in the fp7 project radhar: Objectives and current state," in Proc. of 1st International Conference on Systems and Computer Science (ICSCS), 2012.

[8] A. Kokosy, T. Floquet, G. Howells, H. Hu, M. Pepper, and C. Donz, "SYSIASS An Intelligent Powered Wheelchair," in Proc. of International Conference on Systems and Computer Science (ICSCS2012), 2012.

[9] R. Li, L. Wei, D. Gu, H. Hu, and K. McDonald-Maier, "Multi-layered map based navigation and interaction for an intelligent wheelchair," in Proc. of IEEE International Conference on Robotics and Biomimetics (ROBIO), Dec 2013, pp. 115-120.

[10] R. C. Simpson, "Smart wheelchairs: A literature review." Journal of rehabilitation research and development, vol. 42, no. 4, pp. 423-436, 2004.

[11] C. Urdiales, E. Perez, G. Peinado, M. Fdez-Carmona, J. Peula, R. Annicchiarico, F. Sandoval, and C. Caltagirone, "On the construction of a skill-based wheelchair navigation profile," IEEE Transactions on Neural Systems and Rehabilitation Engineering, vol. 21, no. 6, pp. 917-927, Nov 2013.

[12] S. P. Parikh, V. Grassi, V. Kumar, and J. Okamoto, "Incorporating user inputs in motion planning for a smart wheelchair," in Proc. of IEEE International Conference on Robotics and Automation, vol. 2. IEEE, 2004, pp. 2043-2048.

[13] R. A. Braga, M. Petry, A. P. Moreira, and L. P. Reis, "Intellwheels-a development platform for intelligent wheelchairs for disabled people." in ICINCO-RA (1), 2008, pp. 115-121.

[14] T. Carlson and J. d. R. Millán, "Brain-controlled wheelchairs: a robotic architecture," IEEE Robotics and Automation Magazine, vol. 20, no. EPFL-ARTICLE-181698, pp. 65-73, 2013.

[15] F. Pasteau, M. Babel, and R. Sekkal, "Corridor following wheelchair by visual servoing," in Proc. of IEEE/RSJ Int. Conf. on Intelligent Robots and Systems, Tokyo, Japan, November 2013.

[16] A. Cherubini, F. Chaumette, and G. Oriolo, "Visual servoing for path reaching with nonholonomic robots," Robotica, vol. 29, no. 7, pp. 1037-1048, December 2011.

[17] M. R. Petry, A. P. Moreira, R. A. Braga, and L. P. Reis, "Shared control for obstacle avoidance in intelligent wheelchairs," in Proc. of IEEE Conference on Robotics Automation and Mechatronics (RAM). IEEE, 2010, pp. 182-187.

[18] C. Urdiales, M. Fdez-Carmona, G. Peinado, and F. Sandoval, "Metrics and benchmarking for assisted wheelchair navigation," in Proc. of IEEE International Conference on Rehabilitation Robotics (ICORR), 2013.

[19] L. Demers, R. Weiss-Lambrou, and B. Ska, "The quebec user evaluation of satisfaction with assistive technology (quest 2.0): an overview and recent progress," Technology and Disability, vol. 14, no. 3, pp. $101-105,2002$. 